\title{
Molecular Mechanisms Involved in Yeast Fitness for Ethanol Production
}

\author{
Elis Cristina Araujo Eleutherio*, Fernanda Cigagna Boechat, Rayne Stfhany Silva Magalhães, Germana Breves \\ Rona and Aline Araújo Brasil \\ Department of Chemistry, Federal University of Rio de Janeiro, Brazil
}

Submission: December 01, 2018; Published: February 13, 2019

*Corresponding author: Elis Eleutherio, Department of Chemistry, Federal University of Rio de Janeiro Av Athos da Silveira Ramos, Brazil

\begin{abstract}
Saccharomyces cerevisiae is well adapted to alcoholic fermentation. In this yeast, fermentation predominates over respiration at high glucose concentrations even under the presence of oxygen. Besides the solid fundamental knowledge base in system biology and in yeast metabolic pathways, this microorganism offers many favorable bioprocessing traits which allow robustness under industrial conditions, such as

a. Genetic accessibility

b. High tolerance to fermentative stresses - high temperatures, high ethanol and sugar concentrations, low $\mathrm{pH}$

c. Ideal physiology features (larger cell size, non-pathogenic, short generation time and ability to grow in a highly reproducible and genetically stable way with poor nutrient requirements). In this chapter, we will review the sugar and ethanol metabolism regulation as well as highlight the main molecular mechanism contributors to yeast multiple stress tolerance during fermentation. Furthermore, we will also discuss the future direction of genome and metabolic engineering of yeasts for ethanol production.

Keywords: Ethanol; Glucose; Metabolism; Stress response; Fermentation; Saccharomyces cerevisiae

Abbreviations: PKA: Protein Kinase A; ROS: Reactive Oxygen Species; HSP: Heat Shock Proteins; XR: Xylose Reductase; XDH: Xylitol Dehydrogenase; XI: Xylose Isomerase; ETC: Electron Transport Chain; CAC: Citric Acid Cycle
\end{abstract}

\section{Introduction}

Ethanol is one of the most important fuels in the actuality, especially because through the last years, the worries with the environment are established and growing and the sustainability has been one of the biggest highlights in the industries philosophy. The fossil fuels have been used by humanity for centuries, but it is changing while the concern with the use of theses fuels is growing. That is because fuels, such as oil and coal, are non-renewable and finite, what makes the exploration increasingly harder and more expensive, and is extremely polluting and aggressive to environment. Therefore, the idea of the use of renewable resources that are considered greener, being less aggressive to the environment, is growing. One of the biggest spotlights in this manner, is the production of ethanol which can be performed from renewable sources, is less polluting and can be produced through raw material that are residues of the agroindustry and are, currently, often used as routed to lower value-added applications.

First-generation ethanol is ethanol produced from sugar and starch sources, such as sugarcane and corn. In the case of sugarcane, for example, the plant is processed and the sucrose available in the juice is metabolized to monosaccharaides, glucose and fructose, which are promptly fermented. This process is well es tablished and very viable and the most widely used in terms of ethanol production [1]. However, it has some limitations. One of them is, because it is necessary to plant the raw material for the extraction of sugar and then the fermentation and production of ethanol from it, large tracts of arable soil that could be producing food are used in the production of energy. This is a problem that has been raised for the future, as the world's population is growing a lot and all food production capacity will be needed if the population continues to grow faster than the availability of food. In addition, in the processing of sugarcane for the withdrawal of the juice to produce ethanol, an enormous amount of waste (the sugarcane bagasse), made of cellulose, hemicellulose and lignin, is generated, which is not good for the environment. However, this waste can be harnessed in a more interesting way from the energetic and environmental point of view, by using it to product more ethanol.

Ethanol generated through bagasse and other waste is called second-generation ethanol. This biomass is a much more complex raw material that needs some other treatments before fermentation. A pretreatment is necessary for making carbohydrates more readily available for hydrolysis, which is responsible for 
transforming long chains of carbohydrates into fermentable sugars. The pretreatment may be biological, chemical, mechanical or physical-chemical, while the hydrolysis may be enzymatic or acidic [2]. This is a very interesting process because it adds a value to a waste that would have been destined to a less noble process, such as direct burning for energy, and it is an environmentally friendly strategy. However, like every process, it also has limitations. Currently the pretreatment is usually carried out through physical-chemical treatment (use of acids and temperature, by example) that requires a post-neutralization treatment of the medium so that the subsequent enzymatic treatment for hydrolysis and the fermentation itself does not find a hostile environment and ethanol production can occur.

This process is quite expensive, because there is a high expense with this neutralization besides all the controls that the process implies, like the temperature control. In this respect, an interesting treatment would be biological. However, this treatment is also very expensive due to the conditions that a microorganism demands for it to work optimally, such as $\mathrm{pH}$ and temperature control mainly. Moreover, the availability of sugar in the medium is not simple, since these polymers, especially hemicellulose and mainly lignin, are a complex mixture composed of residues of different monosaccharaides, linked by different glycosidic bonds, which demands action of different microorganisms to treat this structure that is so difficult to break. Many studies have been conducted to reduce these costs in order to increase the feasibility of using these techniques [3]. The best scenario is the production of first-generation ethanol coupled with second-generation ethanol.

\section{The metabolic regulation behind $S$. cerevisiae prefer-} ence for fermentation

The yeast Saccharomyces cerevisiae is the most common microorganism used for alcoholic fermentation in industrial processes [4]. This yeast is one of the simplest eukaryotes and is really versatile, being able to grow at high sugar concentration [5]. The most abundant sugar in nature is glucose, which is also the preferred carbon source of $S$. cerevisiae. Glucose enters the cell through at least 6 transporters (Hxt1, Hxt2, Hxt3, Hxt4, Hxt6, Hxt7) which have different affinities for sugar and have their expressions modulated by the different concentrations of glucose in the medium [6]. The repression of HXT genes occurs in the absence of glucose through the recruitment of the repressor complex Ssn6-Tup1, with the help of Mth1 and Std1 proteins. The HXT genes are induces by glucose though inhibition of Mth1 and Rgt1[6]. Other monosaccharaides may also be fermented by S. cerevisiae, such as mannose and fructose and, after a period of adaptation, galactose. Sucrose is hydrolyzed preferentially by invertase, located at the surface of the cell.

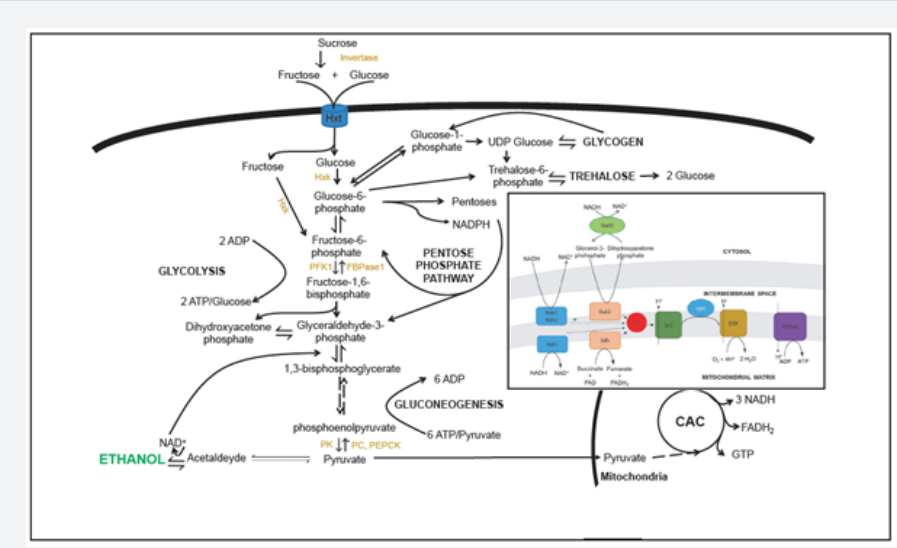

Figure 1: Simplified scheme of ethanol and glucose metabolism. In the figure are represented the pathways that are important for ethanol production and the pathways which regulation is important so ethanol production can be maximized, such as glycolysis, pentose phosphate pathway, gluconeogenesis, glycogen synthesis and degradation and trehalose synthesis and degradation. The inset represents the simplified scheme of ETC.

The cell gain of energy through glucose can occur in two different ways on substrate-level phosphorylation (fermentation) and oxidative phosphorylation (respiration). Once in cytosol, glucose is immediately phosphorylated to glucose-6-phosphate (G6P) to trap sugars inside the cell and avoid diffusion out the cell. G6P is a cell metabolite of the five major yeast glucose pathways glycolysis, gluconeogenesis, glycogen synthesis and degradation, trehalose metabolism and pentose phosphate shunt (Figure 1) [5]. The glycolytic pathway is composed of 10 reactions, producing two ATP molecules, two NADH molecules and two molecules of pyruvate.

Glucose flux is dependent on the activity of enzymes which catalyze irreversible reactions; the other reactions are close to equilibrium and, thus, reversible. The first of those enzymes is hexokinase, which is responsible for the phosphorylation of glucose to glucose-6-phosphate. Unlike other enzymes in the pathway, hexokinase is not regulated by energy demand, as it may give rise to other destinations besides energy production but seems to be inhibited by trehalose-6-phosphate (T6P) [7]. The second enzyme that regulates the glycolytic flux, and the most important one, is phosphofrutokinase1 (PFK1) that converts fructose-6-phosphate to fructose-1,6-bisphosphate. This enzyme is inhibited in an allosteric way by ATP and activated by AMP and fructose-2,6-bisphosphate. Pyruvate kinase is the third enzyme that regulates the glycolytic flux and is also inhibited by ATP [8]. 
Pyruvate can then be directed to two distinct pathways: one oxygen-dependent and one oxygen-independent. If oxygen is present, pyruvate can be transported to the mitochondrial matrix and then converted to Acetyl-CoA, which can be conveyed to the Citric Acid Cycle (CAC; generation of one GTP molecule, three $\mathrm{NADH}$ and one $\mathrm{FADH}_{2}$ ), and then to the Electron Transport Chain (ETC). As electrons are sequentially transferred from NADH/ $\mathrm{FADH}_{2}$ to oxygen, protons are translocated from the matrix to the intermembrane space creating an electrochemical gradient which is used as energy to ATP synthesis. In S. cerevisiae, NADH and $\mathrm{FADH}_{2}$ render 1.5 ATP each one because yeast lacks complex I [9] (Figure 1). Intramitochondrial NADH is reoxidized by an internal NADH ubiquinone oxireduct (Ndi1). Cytosolic NADH can be reoxidized by the external NADH dehydrogenase.

(Nde1/Nde2) or via the glycerol-3-phosphatate dehydrogenase shuttle. Neither Nde1/Nde2 nor Ndi1 are proton pumps [10]. Consequently, in $S$. cerevisiae, the complete oxidation of glucose to $\mathrm{CO}_{2}$ yields 22 ATPs. In the absence of oxygen pyruvate can be directed to the synthesis of ethanol. This synthesis occurs in two reactions conversion of pyruvate to acetaldehyde with the release of $\mathrm{CO}_{2}$, catalyzed by pyruvate decarboxylase enzyme and then from acetaldehyde to ethanol through alcohol dehydrogenase [5]. $S$. cerevisiae has seven different isoforms of alcohol dehydrogenase (Adh1-7p) that is an enzyme responsible for the regeneration of $\mathrm{NAD}+$ cytosolic, that is very important to glycolytic pathway, restoring the redox balance when converts acetaldehyde to ethanol. The isoforms have different characteristics such as their affinities for the substrates and their expressions [11]. What rules if pyruvate will be completely oxidized to $\mathrm{CO}_{2}$ (respiration) or converted into ethanol (fermentation) is not the presence of oxygen but sugar concentration.

Thus, at high-glucose concentration, even when oxygen is present, yeast ferments glucose to ethanol. This is called glucose repression (or Crabtree Effect) and it is a very regulated process. During glucose repression, citric acid cycle and electrons transport chain enzymes are not expressed and other enzymes, responsible for glycolysis, are overexpressed. A few different pathways, signaled by glucose, are responsible for this regulation. Figure $2 \&$ 3 provided visual overview of Snf3/Rgt2-Rgt1, Mig1/Snf1/Hxk2 and Ras/cAMP/PKA pathways in the glucose repression and derepression mechanisms. Although glucose repression has been extensively studied, this mechanism is not completely understood.
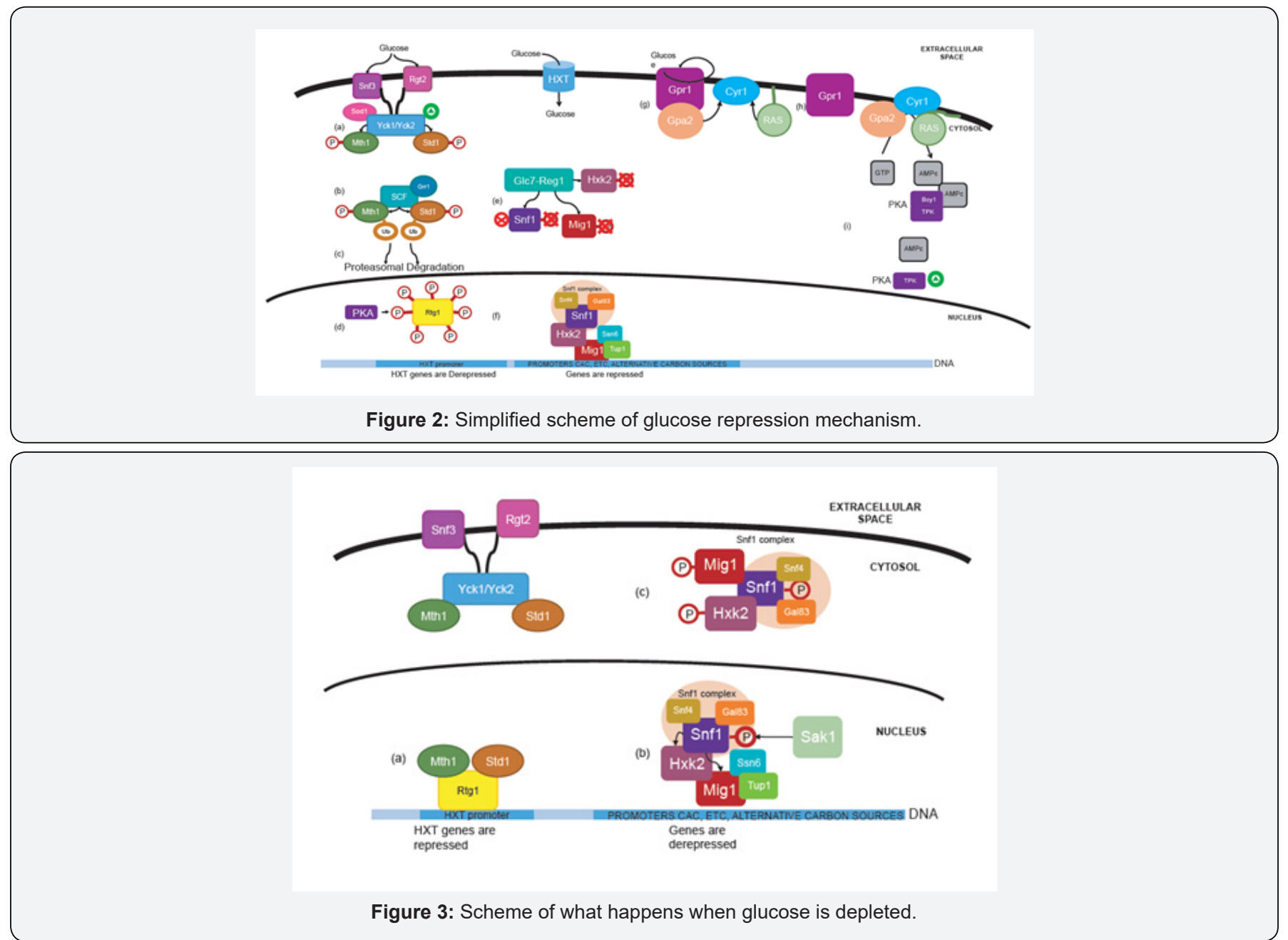

When glucose concentration falls, CAC and ETC enzymes are derepressed, leading to an expressive reduction in the glycolytic flux. The ATP yields obtained from glucose respiration are around 10 times higher from glucose fermentation causing a higher inhi- 
bition of PFK1. By reducing the concentration of intracellular pyruvate, respiration is favored in detriment to the ethanol production since that the overall $\mathrm{Km}$ of mitochondria for pyruvate is of the order of $0.6 \mathrm{mM}$ versus $2.3 \mathrm{mM}$ for the pyruvate decarboxylase I, the key enzyme in alcoholic fermentation $[12,13]$

In order for ethanol production to be as large as possible, it is crucial that substrates of the glycolytic pathway are not diverted to other pathways, thus sugar concentration must be high. At high glucose concentration, gluconeogenesis, glycogen and trehalose synthesis are all impaired [14,15]. Gluconeogenesis is an important pathway responsible to convert pyruvate in G6P. Gluconeogenesis shares with glycolysis the enzymes that catalyze the reactions that are close to equilibrium $(\Delta \mathrm{G} \sim 0)$, and it has its regulation through the other to enzymes, fructose-1,6-bisphosphatase converts fructose-1,6-phosphate in fructose-6-phosphate [16]. As expected, the regulation of gluconeogenesis is contrary to what occurs in glycolysis, that is, the ATP, in this case, is an activator. Besides, as mentioned, gluconeogenesis flux is also regulated by other mechanisms, such as the downregulation of fructose-1,6-bisphosphatase and the upregulation of phosphofructokinase I by PKA phosphorylation [16].

The Snf3/Rgt2-Rgt1 pathway is responsible for the regulation of the expression of glucose transporters genes, regulating the glucose uptake [17]. Glucose binding to Rtg2 and Snf3, activates Yck1 and Yck2, which, in turn, phosphorylate Mth1 and Std1 (2a); Yck1 and Yck2 are stabilized by Sod1[18]. In the phosphorylated form, Mth1 and Std1 are recognized by SCFGrr1 ubiquitin ligase and, consequently, are ubiquitinated ( $2 \mathrm{~b}$ ), becoming a target for proteasomal degradation (2c). Without Mth1 and Std1, Rgt1 is hyperphosphorylated by Protein Kinase A (PKA) leading to HXT genes derepression increasing intracellular concentration of the glucose $[17,19](2 \mathrm{~d})$. Another important pathway to glucose repression is Mig1/Snf1/Hxk2 pathway. At high-glucose levels, Hxk2, Mig1 and Snf1 are dephosphorylated by Glc7-Reg1 protein phosphatase (2e). In this form, Snf1 is inhibited and Mig1 and Hxk2 are in the nucleus, where a repression complex is formed. Ssn6 and Tup1 are co-repressors [20]. Hxk2 binds to Mig1, stabilizing the association of Mig1 with the target gene promoter [21].

The repression complex is also formed by Snf1 complex, which include Snf1, Snf4, and Gal83; Snf1complex is linked to the repression complex through the binding of Snf1 to Hxk2 [21] (2f). Genes which codes for enzymes of CAC, ETC, alternative carbon sources consumption and gluconeogenesis are targets of glucose repression complex [20]. Other pathway involved with glucose repression is the Ras/cAMP/PKA pathway. Glucose interacts with Gpr1, activating the G proteins Gpa2 and Ras (2g), which interact with adenylate cyclase (Cyr1) stimulating cAMP synthesis [22,23] (2h). cAMP binds to the regulatory Bcy1 subunits of PKA, causing the dissociation of the regulatory subunits from the catalytic subunits (TPK), activating PKA [23] (2i). PKA has an important role because it is responsible for the phosphorylation, and thus regulation, of a lot of the enzymes involved in glucose signaling, such as Rgt1 that was previously quoted [19]. Other PKA targets are phosphofructokinase 2, fructose-1,6-bisphosphatase, glycogen synthase, glycogen phosphorylase and threhalase, impairing gluconeogenesis, trehalose and glycogen synthesis and favoring glycolysis [24].

Mth1 and Std1 are recruited, causing a conformation change that makes possible the binding of Rgt1 to its recognition sites in DNA generating transcriptional repression [23] (3a). At low-glucose concentration, Snf1 is phosphorylated, and activated by Sak1; in this form, Snf1 phosphorylates Hxk2 and Mig1 (3b), disassembling the repression complex in the nucleus and leading to Mig1/Hxk2 exportation to the citosol [21] (3c).

\section{Stress response during alcoholic fermentation in S. cer- evisiae}

The ability of $S$. cerevisiae to overcome other microorganisms and dominates the culture medium during alcoholic fermentation is associated to its high fermentative performance and capacity to withstand the adverse conditions of fermentation processes [25]. During fermentation, cells are submitted to different kinds of stress, such as accumulation of ethanol, high osmolarity, heat and oxidative stress. These stresses can occur in concert or sequentially and have different impacts on yeast cells. The ability of a given yeast strain to respond to these stresses determines its robustness and its performance in industrial processes.

\section{Ethanol stress}

Ethanol is the final and interest product of fermentation process and also the main stress factor for yeast cells [26]. Even at low concentration, ethanol inhibits cell division, decreasing the growth rate due to the inhibition of glucose and amino acids consumptions [27]. The impact is higher at the end of the fermentation. As ethanol concentration increase, reaching high levels, the membrane permeability is altered, which increases the influx of protons (lowing the $\mathrm{pH}$ at the cytosol to toxic levels); proteins are denatured, impairing their functions in cell metabolism; the levels of Reactive Oxygen Species (ROS) increase, leading to an oxidative stress; and the water availability decreases, causing dehydration $[27,28]$. All of them impact on cell viability, growth rate and ethanol yield [29].

The main targets of ethanol are membranes and proteins, influencing their structure and functions [28]. Ethanol interacts with the lipid bilayer of the plasma membrane through the hydrophilic side, disturbing the original membrane structure $[27,30]$. The fluidity and integrity of the plasma membrane are important for cell protection; therefore, it is common to detect high levels of expression of genes responsible for ergosterol synthesis [5] and structure organization and biogenesis of cell wall [31]. Ethanol also interacts with proteins, forming a H-bond with the hydrophilic residues of the proteins, destroying the bond patterns, leading to protein denaturation $[27,32]$. As proteins are denatured, they loss their function, which is harmful for different metabolic pathway into the cells. One of the main protein targets of ethanol are 
glycolytic enzymes, such as hexokinase and pyruvate kinase, impacting directly the fermentation rate [27].

As yeast cells are submitted to ethanol stress, some biochemical mechanisms are activated to improve tolerance against the stressful situation. The most common mechanism that can be detected in yeast cells under heat and ethanol stress are trehalose synthesis and expression of Heat Shock Proteins (Hsps) [31]. The disaccharide trehalose is synthetized in a wide range of organisms, among them yeast, mainly as a response to several stress conditions [33]. Some experiments have shown that the levels of trehalose increase during fermentation, indicating that the mechanism is activated to confer tolerance to the cell in such condition [34]. In addition, it was observed that mutant strains unable to hydrolyze trehalose show increased tolerance to ethanol stress, confirming the importance of this disaccharide to confer robustness during fermentation [35]. This sugar mitigates the denaturing effects of ethanol chaotropicity by stabilizing the structure of membranes and proteins [36]. Trehalose avoids denaturation by excluding water from protein or membranes' hydration layer and ordering around it. Trehalose-water interaction is stronger than water-water. At high concentration, trehalose competes against biomolecule for the available water, restricting the mobility of the water molecules of the hydration layer which stabilizes biomolecule structure during stress.

S. cerevisiae cells also synthesize HSPs for protection against ethanol stress, in the same way that is observed in heat shock. Among the 6 different Hsps analyzed and related to ethanol stress, only Hsp104 and Hsp12 have been related to tolerance to this condition [28]. Hsp104 does not avoid protein denaturation, differently from other chaperones, but preserve protein structures, allowing their reactivation after the end of the stress [37]. It was observed that mutant strains that do not express Hsp12 show low tolerance to ethanol stress [38]. It has been shown that this chaperone is localized only at the plasma membrane, suggesting that Hsp12 is involved with membrane protection.

\section{Oxidative stress}

Although ethanol production by $S$. cerevisiae is an anaerobic process, cells need $\mathrm{O}_{2}$ to grow in optimal conditions in the first steps of fermentation and increase ethanol yield. Due this, it is common to name the fermentation as a semi anaerobic process, and, thus, passable to be submitted to oxidative stress [27]. Oxidative stress is characterized by the imbalance between the antioxidant response and $\mathrm{ROS}\left(\mathrm{O}_{2}, \mathrm{OH}\right.$ and $\left.\mathrm{H}_{2} \mathrm{O}_{2}\right)$ [39]. When this happens, the ROS levels increase, inducing negative effects into the cells as damage at the membrane, lipids, proteins, DNA; as a consequence, cell growth is inhibited, and apoptosis is activated [40].

During fermentation, ethanol stress and heat shock can induce an increase in ROS levels or inactivation of antioxidant enzymes $[41,42]$. Even under anaerobic fermentations, some metabolic pathways require the presence of molecular oxygen; ROS can be produced by NAD(P)H-dependent pathways, such as cytochrome P450 systems [43]. Ethanol reduces biomolecules hydration layer, making them more prone to ROS attack. According to Trevisol et al. [35], yeast cells showed increased levels of lipid peroxidation and protein oxidation after fermentation. ROS can also be produced during the pretreatment process required for the use of lignocellulosic residues in second generation ethanol [44].

\section{Some studies have shown that strains used to produce bioethanol show high levels of ROS, as well as the up- regulation of some antioxidant compounds}

a. The enzymes $\mathrm{Cu} / \mathrm{Zn}$ superoxide dismutase, which dismutates $\mathrm{O}_{2}$ - to $\mathrm{H}_{2} \mathrm{O}_{2}$ and $\mathrm{O}_{2}$, and catalase, which converts $\mathrm{H}_{2} \mathrm{O}_{2}$ to water and $\mathrm{O}_{2}$

b. glutathione $[27,39]$. When these defenses are not working, the cells lose the ability to grow, then decreasing the fermentation rate, that means, drop of ethanol production.

In industrial processes, yeast cells are recycled and reused in serial fermentation batches, leading to cell aging and increasing petite concentration which reduces ethanol yields [35]. More stable mitochondria are essential for a better protection against ROS. Overexpression of mitochondrial cytochrome $\mathrm{C}$ oxidase chaperone gene (COX20) improved oxidative, acid and ethanol stress tolerance and increased ethanol yield [44].

Competition assays coupled to quantitative proteomic analysis revealed that the improved fermentation traits of the dominant strain is linked to increased levels of proteins involved in response to oxidative stress, such as Sod1 and Trx1, and trehalose synthesis [45]. On the other hand, mutants unable to express Sod1 or Trx1 or to synthesize trehalose showed an impaired fermentation performance, confirming that the abilities of accumulating high levels of trehalose and coping with oxidative stress are crucial for improving fermentation.

\section{Heat stress}

Both first- and second-generation ethanol production use high temperatures in the fermentation of $S$. cerevisiae [46]. Fermentation process for bioethanol production at high temperatures presents some financial advantages. High temperatures avoid contamination, reduce cooling costs and allow simultaneous saccharification and fermentation (this is interesting when ethanol is produced from corn) $[47,48]$. On the other hand, high temperatures directly affect yeast growth rate and lead to protein denaturation [49]. Yeast cultivation under increased temperatures leads to petite mutation, which also impairs fermentation $[35,50]$.

Heat stress enhance the H+-ATPase activity, protein responsible for the active transport of protons across the membrane, leading to an increase in the output of protons from the cell and membrane depolarization [50]. Heat shock causes protein denaturation, besides causing a disorder in the plasma membrane, increasing membrane permeability [50].

Response to thermal stress is activated at temperatures above $35^{\circ} \mathrm{C}$, inducing heat shock proteins [50]. Hsp proteins play an important role in folding and refolding proteins, as well as de- 
grading misfolding and denatured proteins [51]. The major Hsp involved in the defense against heat shock are Hsp70 and Hsp104. Hsp 70 is involved in the response to thermal stress acting in the aggregate's prevention and proteins refolding [51]. Hsp104 has a unique characteristic, it rescues inactivated proteins from insoluble aggregates, formed by thermal stress, then these proteins are refolded by other chaperones like Hsp70 [51].

During thermal stress there is an enhance in the antioxidant enzymes activity due to increased production of ROS, raising levels of mitochondrial manganese superoxide dismutase (SOD2 gene) [50], cytosolic copper-zinc Superoxide Dismutase (SOD1 gene) [52] and cytosolic catalase T (CTT1 gene) [50]. Other way of yeast cell defends itself against thermal stress is by producing trehalose. It was observed that genes involved in the cytoskeleton, such as SAC6, SHE4, SLA2, SPC72, are required against heat stress [52]. Other genes were demanded for thermal tolerance like genes involved in transcription (such as MED1, MED2, PAF1, SWI3, SWI6 and SRB2), RNA processing (such as ISY1, LEA1, LSM6) and actin cytoskeleton (such as SAC6, SHE4, SLA2, SPC72) [52].

\section{Osmotic stress}

The large amount of sugar found in the fermentation medium to enhance fermentation rate leads to osmotic stress $[47,49,52]$ This stress leads to a contraction of the cell due to cytoplasmatic water waste, leading to loss of turgor pressure [27,51,52]. In order to balance the osmotic pressure across the membrane the yeast cell produces glycerol, a compatible osmolyte $[27,47,52]$, by the HOG- MAPK pathway (high-osmolarity glycerol-mitogen-activated protein kinase) [52]. Vacuolar protein transport genes, such as VPS1, VPS3, VPS16, genes involved in energy metabolism, such as OXA1, PET100, and SCO1, and cell defense genes such as HOG1 and SOD1, are required in osmotic stress [52].

Msn2 and Msn4 are binding STRE (stress response element) factors involved in the stress response against osmotic stress. Msn2 and Msn4 have an indirect protective role in Hog1 against specific phosphatases in the nucleus [51]. HOG pathway induces GPD1 and GPP2, under osmotic stress, leading to glycerol production [51]. Gpd1 (glycerol-3-phosphate dehydrogenase) catalyze the conversion of dihydroxyacetone phosphate to glycerol-3-phosphate (G3P), and Gpp2 (glycerol-3-phosphate phosphatases hydrolizes G3P into glycerol [51]. After osmotic shock, the membrane protein Fps1 is closed accumulating glycerol inside the cell [51].

\section{pH stress}

Lignocellulosic hydrolysate of second-generation ethanol fermentation contains furans derivatives and weak acids, which impair fermentation [53]. In first generation ethanol fermentation process, yeast cells must be washed with sulfuric acid in order to avoid contamination by bacteria before be reused in another batch. In that way, yeast can last up to 6 months if no contamination occurs [54]. This process reduces cell viability and ethanol yield [55]. The pH medium affects yeast growth rate, fermentation products $[49,53,56]$, inhibits glycolytic flux and induces oxidative stress [53]. The permeability of some nutrients is dependent on the concentration of $\mathrm{H}+$ in the culture medium [49].

Weak acids have inhibitory effect on cell growth, owing to the fact of non-dissociated acids cross the plasma membrane through passive transport, decreasing cytoplasmatic $\mathrm{pH}[56,57]$. There are two hypotheses to explain the effect of weak acids: intracellular concentration of anions and decoupling [56]. By the decoupling theory, the decrease in intracellular $\mathrm{pH}$ leads to a pumping of protons out of the cell by ATPase, plasma membrane, expending ATP [56]. The response to weak acids is dependent on the HOG-MAPK pathway and pathways related to cell wall synthesis [58,59]. The response to inorganic acids is similar to response to weak acids. However, inorganic acid tolerance relies on a mechanism Protein Kinase A (PKA) dependent [58]. In acidic media the yeast activates the general stress response to adapt, already stress tolerance involves the regulation of the cell cycle, decreasing transcription levels and protein synthesis [58]. YGP1, TPS1 and HSP150 are induced in $\mathrm{pH}$ stress [60]. It was observed a low increase in trehalose concentration under acid stress in S. cerevisiae [53]. Although yeast develops some defense mechanisms, engineering a low $\mathrm{pH}$ tolerant cell is one of the targets of second-generation ethanol synthesis [61].

\section{S. cerevisiae in sustainable fermentation: development of strains for second generation ethanol}

The sustainable bioethanol production from lignocellulosic residues has been largely studied worldwide due to its considerable amount of potentially fermentable sugars [62]. The main structural components of Lignocellulosic Biomass (LCB), such as woods and agricultural residues, are cellulose, hemicelluloses and lignin. To produce ethanol by fermentation of complex polysaccharides (cellulose and hemicelluloses), different pretreatment methods including chemical and enzymatic hydrolysis are used to convert it into simple monosaccharides. Typically, lignocellulosic hydrolysates contain both pentose sugars, such as D-xylose and L-arabinose as well as hexose sugars. The main component of lignocellulosic hydrolysates is glucose (60-70\%), and the second most abundant carbohydrate is D-xylose, consisting $30-40 \%$ of the cellulosic hydrolysates $[63,64]$. Thus, the development of economically feasible lignocellulosic ethanol production also depends on the utilization of xylose.

Hexoses are nicely fermented by $S$. cerevisiae, but not xylose [65]. Several microorganisms are able to ferment xylose, but none shows the interesting features to alcoholic fermentation as many as this yeast high ethanol productivity, multiple stress tolerance and resistance to inhibitory compounds present in the hydrolysate of lignocellulosic biomass [66]. Numerous studies have attempted different metabolic engineering strategies to overcome the restrictions of xylose metabolism and improve the xylose fermentation performance of $S$. cerevisiae $[67,68]$. 
Xylose-fermenting microorganisms use two different pathways to isomerize xylose into xylulose: the balanced-redox oxidoreductase and the isomerase pathway. The first one consists of two sequential enzymatic reactions: conversion of xylose into xylitol via Xylose Reductase (XR) followed by conversion of xylitol into xylulose via Xylitol Dehydrogenase (XDH); both enzymes use the same cofactor, avoiding redox unbalance. On the other hand, the Xylose Isomerase (XI) pathway, found in some bacteria and fungi, isomerizes directly xylose into xylulose without a cofactor requirement [69]. Both the oxidoreductase and the isomerase pathways have been favorably introduced into $S$. cerevisiae, enabling recombinant strains to produce ethanol from xylose [70].

By comparing the xylose-fermenting abilities between a $\mathrm{XI}$-expressing recombinant $S$. cerevisiae strain and a XR-XDH-expressing strain, the first one has shown the lowest xylitol yield and the highest ethanol yield [71]. However, XI-expressing strains showed a much lower xylose fermentation rate. High ethanol yield and productivity were only achieved when XI expression was under strong promoters in multicopy plasmids, suggesting that high XI activities are necessary for efficient xylose fermentation. The cloning of XI from different microorganisms, Piromyces sp [16], Clostridium phytofermentans [72], Orpinomyces sp [73], Prevotella ruminicola [69], Burkholderia cenocepacia [74], produced high XI activity in the recombinant yeast strains.

Some additional genetic modifications were necessary to enhance the performance of recombinant yeasts which isomerize xylose into xylulose. The overexpression of the endogenous XKS1(codes for xylulokinase), the deletion of GRE3 (codes for a reductase able to convert xylose into xylitol using NADPH) together with the overexpression of all non-oxidative phosphate pentose pathway - PPP - enzymes, Tal1, Tkl1, Rpe1, Rki1, improved growth on xylose and xylose fermentation in a strain carrying a bacterial XI [75] and also in a strain carrying Piromyces XI [71]. Both the requirement of PPP activity for xylose metabolism and the increase in the activity to convert xylose to xylulose seems to be crucial for xylose fermentation in S. cerevisiae. Subsequently, the relevance of high PPP activity in xylose-utilizing $S$. cerevisiae was confirmed by metabolic flux analysis [76] as well as by microarray analysis [77]

Due to the absence of xylose-specific transporters, S. cerevisi$a e$ has been engineered for xylose utilization. This microorganism assimilates xylose by facilitated diffusion mainly through non-specific hexoses transporters encoded by HXT genes [78]. However, in ethanol producing strains, the xylose transport is limited in the presence of glucose and other pentose sugars through competitive inhibition during co-fermentation of glucose and xylose [79]. Xylose transporters derived from distinct xylose utilizing microorganisms have been cloned into yeast strains. Furthermore, the alteration of xylose interacting motif as well as the hexose transporters engineering have been also used to improve xylose transportation [80].

S. cerevisiae harboring XYL1 gene from $S$. stipitis increased the xylose uptake about 7.9-folds on supplementing $20 \mathrm{~g} \mathrm{~L}^{-1}$ glucose
[81]. In addition, the authors have shown an increased expression level of HXT4 and HXT7 genes, which encodes for Hxt4p and Hxt7p transporter proteins [81]. It has been also demonstrated that both genes designated GXF1 (glucose/xylose facilitator 1) and GXS1 (glucose/xylose symporter 1) have been expressed in $S$. cerevisiae [82], and the recombinant Gxf1- expressing S. cerevisiae strain has shown faster xylose uptake and ethanol production [83]. Recently, the incorporation of a transporter gene MGT05196 (xylose transport from Meyerozyma guilliermondii) into S. cerevisiae, showed a 26.3-fold higher growth as compared to wild-type strain [84]. Furthermore, it has been suggested that a mutation at N360F of MgT05196 enhances the D-xylose transport activities without any glucose-inhibition [84]. Thus, engineering specific xylose transporters without glucose inhibition, or overcoming the metabolic inhibition is essential for further improving the development of glucose-xylose co-fermentation strains for biomass refining.

According to some authors, glucose repression is a barrier for the use of xylose in lignocellulosic hydrolysates [85]. In contrast, Vilela et al. [86] demonstrated that the xylose consumption during fermentation of a glucose-xylose blend was improved when yeast cells expressing Burkholderia cenocepacia XI were previously grown on glucose, as opposed to xylose alone [86]. Xylose-grown cells show increased expression of Mig1 repressed genes [87], as Mig1 is dephosphorylated and active only at high glycolytic rates [21] Thus, when growing on xylose, yeast cells switch the mode of metabolism from fermentation to respiratory, reducing the glycolytic flux which is detrimental for ethanol yield and productivity. Thus, the detrimental effect of glucose over xylose consumption seems not to be associated to catabolite repression.

Vilela et al. [86] used evolutionary engineering to improve xylose fermentation by the recombinant yeast expressing Burkholderia cenocepacia XI, which involved sequential batch cultivation on xylose [86]. This strategy has been applied to increase the ethanol yield and productivity of xylose-fermenting recombinant yeast strains [30]. The improvement in xylose fermentation showed by the Burkholderia cenocepacia XI recombinant strain submitted to evolutionary engineering was associated to the increase in the expression of HXT2 and TAL1 genes, which code for a low-affinity hexose transporter and transaldolase, respectively

[86]. The use of xylose instead of glucose has several effects on the yeast metabolome that are specific to anaerobic consumption of xylose. For example, the reaction catalyzed by Tal1 is a rate-limiting step for the conversion of xylose into ethanol [88]. Hxt2 has the second highest transport capacity, taking up xylose at a rate of $8.74 \mathrm{~g} / \mathrm{h} / \mathrm{g}$ dry weight of cell at high sugar concentrations. Therefore, it should be expected a positive effect on xylose utilization under increased HXT2 and TAL1 expressions. Taken together, the results obtained by Vilela et al. [86] show that is necessary to understand more deeply the metabolic regulation of xylose-ethanol conversion yeast to increase the efficiency of fermentation [86]. Many works have tried to increase the flux of the xylose-ethanol 


\section{Advances in Biotechnology \& Microbiology}

pathway in Saccharomyces cerevisiae by raising the supply of the enzymes. However, the activity of the enzymes depends on the environmental and intracellular conditions. This means that is necessary to understand how cells control their metabolism.

Currently, some of the most powerful tools for targeting metabolic changes to improve the xylose fermentation are functional genomics, including the transcriptome, proteome, metabolome and fluxome. These are emerging areas of future research for enhancing the rate and yield of ethanol production from xylose. Microarray technology as well as CRISPR/Cas9 technique have been also gradually used in xylose-metabolizing recombinant strains, for global expression studies and to improve xylose utilization pathways, respectively, providing important advances on xylose fermentation [87]. Although there still remain challenges in ethanol production from xylose using metabolically engineered $S$. cerevisiae, the combination of metabolic engineering with functional genomics analysis and/or evolutionary approaches may open novel avenues for developing strategies to an effective xylose fermentation as well as ethanol production.

\section{References}

1. Goldemberg J, Guardabassi P (2010) The potential for first-generation ethanol production from sugarcane. Biofuels Bioprod Biorefining 4(1): $17-24$.

2. Aditiya HB, Mahlia TMI, Chong WT, Nur H, Sebayang AH (2016) Second generation bioethanol production: A critical review. Renew Sustain Energy Rev 66: 631-653.

3. Zabed H, Faruq G, Sahu JN, Azirun MS, Hashim R, Boyce AN (2014) Bioethanol Production from Fermentable Sugar Juice, 2014: 11.

4. Tesfaw A, Assefa F (2014) Current trends in bioethanol production by Saccharomyces cerevisiae: substrate, inhibitor reduction, growth variables, coculture, and immobilization. Int Sch Res Not 2014: 532852.

5. Wills C (1990) Regulation of Sugar and Ethanol Metabolism in Saccharomyces cerevisiae. Crit Rev Biochem Mol Biol 25(4): 245-280.

6. Kim JH, Roy A, Jouandot D, Cho KH (2013) The glucose signaling network in yeast. Biochim Biophys Acta 1830(11): 5204-5210.

7. Blázquez MA, Lagunas R, Gancedo C, Gancedo JM (1993) Trehalose-6-phosphate, a new regulator of yeast glycolysis that inhibits hexokinases, FEBS Lett 329(1-2): 51-54.

8. Larsson C, Påhlman IL, Gustafsson L (2000) The importance of ATP as a regulator of glycolytic flux in Saccharomyces cerevisiae. Yeast 16(9): 797-809.

9. Herrero E, Ros J, Bellí G, Cabiscol E (2008) Redox control and oxidative stress in yeast cells, Biochim Biophys Acta 1780(11): 1217-1235.

10. de Vries S, Marres CA (1987) The mitochondrial respiratory chain of yeast. Structure and biosynthesis and the role in cellular metabolism. Biochim Biophys Acta 895(3): 205-239.

11. De Smidt 0, du Preez JC, Albertyn J (2008) The alcohol dehydrogenases of Saccharomyces cerevisiae: A comprehensive review, FEMS Yeast Res 8(7): 967-978.

12. Sergienko EA, Jordan F (2001) Catalytic Acid-Base Groups in Yeast Pyruvate Decarboxylase. 2. Insights into the Specific Roles of D28 and E477 from the Rates and Stereospecificity of Formation of Carboligase Side Products. Biochemistry 40 (6): 7369-7381.

13. Pronk JT, Yde Steensma H, Van Dijken JP (1996) Pyruvate metabolism in Saccharomyces cerevisiae. Yeast 12(16): 1607-1633.
14. François J, Parrou JL (2001) Reserve carbohydrates metabolism in the yeast Saccharomyces cerevisiae, FEMS Microbiol Rev 25 (1): 125-145.

15. Trevisol ET, Panek AD, De Mesquita JF, Eleutherio EC (2014) Regulation of the yeast trehalose-synthase complex by cyclic AMP-dependent phosphorylation. Biochim Biophys Acta 1840(6): 1646-1650.

16. Müller D, Exler S, Aguilera-Vázquez L, Guerrero-Martín E, Reuss M (2003) Cyclic AMP mediates the cell cycle dynamics of energy metabolism in Saccharomyces cerevisiae. Yeast 20(4): 351-367.

17. Snowdon C, Johnston M (2016) A novel role for yeast casein kinases in glucose sensing and signaling. Mol Biol Cell 27(21): 3369-3375.

18. Tsang CK, Liu Y, Thomas J, Zhang Y, Zheng XF (2014) Superoxide dismutase 1 acts as a nuclear transcriptional factor to regulate oxidative stress resistance. Nat Commun 5: 3446 .

19. Kayikci Ö, Nielsen J (2015) Glucose repression in Saccharomyces cerevisiae. FEMS Yeast Res. 15(6): pii: fov068.

20. Raghevendran V, Nielsen J, Olsson L (2005) Teaching microbial physiology using glucose repression phenomenon in Baker's yeast as an example. Biochem Mol Biol Educ 33(6): 404-410.

21. Vega M, Riera A, Fernández-Cid A, Herrero P, Moreno F (2016) Hexokinase 2 Is an intracellular glucose sensor of yeast cells that maintains the structure and activity of mig1 protein repressor complex. J Biol Chem 291(14): 7267-7285.

22. Toda T, Uno I, Ishikawa T, Powers S, Kataoka T, et al. (1985) In Yeast, RAS proteins are cotrolling elements of adeylate cyclase. Cell 40(1): 27-36.

23. Santangelo GM (2006) Glucose Signaling in Saccharomyces cerevisiae. Microbiol Mol Biol Rev 70(1): 253-282.

24. Thevelein JM, de Winde JH (1999) Novel sensing mechanisms and targets for the cAMP-protein kinase A pathway in the yeast Saccharomyces cerevisiae, Mol Microbiol 33(5): 904-918.

25. Albergaria H, Arneborg N (2016) Dominance of Saccharomyces cerevisiae in alcoholic fermentation processes: role of physiological fitness and microbial interactions. Appl Microbiol Biotechnol 100 (5): 20352046.

26. Carmona-Gutierrez D, Sommer C, Andryushkova A, Kroemer G, et al. (2012) A higher spirit: avoiding yeast suicide during alcoholic fermentation. Cell Death Differ 19(6): 913-914.

27. C Auesukaree (2017) Molecular mechanisms of the yeast adaptive response and tolerance to stresses encountered during ethanol fermentation. J Biosci Bioeng 124(2): 133-142.

28. Stanley D, Bandara A, Fraser S, Chambers PJ, Stanley GA (2010) The ethanol stress response and ethanol tolerance of Saccharomyces cerevisiae, J Appl Microbiol 109(1): 13-24.

29. Kim IS, Kim YS, Kim H, Jin I, Yoon HS (2013) Saccharomyces cerevisiae KNU5377 stress response during high-temperature ethanol fermentation. Mol Cells 35(3): 210-218.

30. Ding J, Huang X, Zhang L, Zhao N, Yang D (2009) Tolerance and stress response to ethanol in the yeast Saccharomyces cerevisiae. Appl Microbiol Biotechnol 85(2): 253-263.

31.Zhao XQ Bai FW (2009) Mechanisms of yeast stress tolerance and its manipulation for efficient fuel ethanol production. J Biotechnol 144 (1): $23-30$.

32. Kundu S, Aswal VK, Kohlbrecher J (2017) Effect of ethanol on structures and interactions among globular proteins. Chemical Physics Letters 670: 71-76

33. Eleutherio E, Panek A, De Mesquita JF, Trevisol E, Magalhães R (2015) Revisiting yeast trehalose metabolism. Curr Genet 61 (2015) 263-274. 


\section{Advances in Biotechnology \& Microbiology}

34. Jun Shima, Akihiro Hino, Chie Yamada-Iyo, Yasuo Suzuki, Ryouichi Nakajima, et al. (1999) Stress tolerance in doughs of Saccharomyces cerevisiae trehalase mutants derived from commercial Baker's yeast. Appl Environ Microbiol 65: 2841-2846.

35. Trevisol ET, Panek AD, Mannarino SC, Eleutherio EC (2011) The effect of trehalose on the fermentation performance of aged cells of Saccharomyces cerevisiae. Appl Microbiol Biotechnol 90(2): 697-704.

36. Cray JA, Stevenson A, Ball P, Bankar SB, Eleutherio EC, et al. (2015) Chaotropicity: a key factor in product tolerance of biofuel-producing microorganisms. Curr Opin Biotechnol 33: 228-259.

37. Glover JR, Lindquist S, Hsp 10, Hsp70, and Hsp40: a novel chaperone system that rescues previously aggregated proteins. Cell 94(1): 73-82.

38. Sales K, Brandt W, Rumbak E, Lindsey G (2000) The LEA-like protein HSP 12 in Saccharomyces cerevisiae has a plasma membrane location and protects membranes against desiccation and ethanol-induced stress. Biochim Biophys Acta 1463 (2): 267-278.

39. Eleutherio E, Brasil AA, França MB, de Almeida DSG, Rona GB, et al. (2018) Oxidative stress and aging: Learning from yeast lessons. Fungal Biol 122(6): 514-525.

40. Sasano Y, Haitani Y, Hashida K, Ohtsu I, Shima J (2012) Enhancement of the proline and nitric oxide synthetic pathway improves fermentation ability under multiple baking-associated stress conditions in industrial baker's yeast. Microb Cell Fact 11: 40 .

41. Landolfo S, Politi H, Angelozzi D, Mannazzu I (2008) ROS accumulation and oxidative damage to cell structures in Saccharomyces cerevisiae wine strains during fermentation of high-sugar-containing medium. Biochim Biophys Acta 1780 (6): 892-898.

42. Santiard D, Ribiére C, Nordmann R, Houee-Levin C (1995) Inactivation of $\mathrm{Cu}, \mathrm{Zn}$-superoxide dismutase by free radicals derived from ethanol metabolism: a gamma radiolysis study. Free Radic Biol Med 19: 121 127.

43. Merico A, Sulo P, Piskur J, Compagno C (2007) Fermentative lifestyle in yeasts belonging to the Saccharomyces complex. FEBS J 274(4): 976989

44. Kumar V, Hart AJ, Keerthiraju ER, Waldron PR, Tucker GA, et al. (2015) Expression of Mitochondrial Cytochrome C Oxidase Chaperone Gene (COX20) Improves Tolerance to Weak Acid and Oxidative Stress during Yeast Fermentation. PLoS One 10(10): e0139129.

45. Santos RM, Nogueira FC, Brasil AA, Carvalho PC, Leprevost FV, et al (2017) Quantitative proteomic analysis of the Saccharomyces cerevisiae industrial strains CAT-1 and PE-2. J Proteomics 151: 114-121.

46. Nair RB, Kalif M, Ferreira JA, Taherzadeh MJ, Lennartsson PR (2017) Mild-temperature dilute acid pretreatment for integration of first- and second-generation ethanol processes. 245(ptA): 145-151.

47. Kitichantaropas Y, Boonchird C, Sugiyama M, Kaneko Y, Harashima S, Cellular mechanisms contributing to multiple stress tolerance in Saccharomyces cerevisiae strains with potential use in high-temperature ethanol fermentation. AMB Express 6(1): 107.

48. Abdel-Banat BM, Hoshida H, Ano A, Nonklang S, Akada R (2010) High-temperature fermentation: how can processes for ethanol production at high temperatures become superior to the traditional process using mesophilic yeast? Appl Microbiol Biotechnol 85(4): 861867

49. Mohd Azhar SH, Abdulla R, Jambo SA, Marbawi H, Gansau JA, et al. (2017) Yeasts in sustainable bioethanol production: A review. Biochem Biophys Rep 10: 52-61.

50. PW Piper (1995) The heat shock and ethanol stress responses of yeast exhibit extensive similarity and functional overlap. FEMS Microbiol Lett 134(2-3): 121-127.
51. Estruch F (2000) Stress-controlled transcription factors, stress-induced genes and stress tolerance in budding yeast. FEMS Microbiol Rev 24(4): 469-486

52.Auesukaree C, Damnernsawad A, Kruatrachue M, Pokethitiyook P, Boonchird C, et al. (2009) Genome-wide identification of genes involved in tolerance to various environmental stresses in Saccharomyces cerevisiae. J Appl Genet 50(3): 301-310.

53. Guo ZP, Olsson L (2016) Physiological responses to acid stress by Saccharomyces cerevisiae when applying high initial cell density. FEMS Yeast Res 16(7): fow072.

54. Basílio AC, de Araújo PR, de Morais JO, da Silva Filho EA, et al (2008) Detection and Identification of Wild Yeast Contaminants of 17 the Industrial Fuel Ethanol Fermentation Process. Curr Microbiol 56(4): 322-326.

55. Brosnan MP, Donnelly D, James TC, Bond U (2000) The stress response is repressed during fermentation in brewery strains of yeast. J Appl Microbiol 88(5): 746-755.

56. Palmqvist E, Hahn-Hägerdal B (2000) Fermentation of lignocellulosic hydrolysates. II: inhibitors and mechanisms of inhibition. Bioresource Technology 74(1): 25-33.

57. Kolaczkowska A, Goffeau A (1999) Regulation of pleiotropic drug resistance in yeast. Drug Resist Updat 2(6): 403-414.

58. de Melo HF, Bonini BM, Thevelein J, Simões DA, Morais MA (2009) Physiological and molecular analysis of the stress response of Saccharomyces cerevisiae imposed by strong inorganic acid with implication to industrial fermentations. J Appl Microbiol 109(1): 116-127.

59. Mollapour M, Fong D, Balakrishnan K, Harris N, Thompson S, et al. (2004) Screening the yeast deletant mutant collection for hypersensitivity and hyper-resistance to sorbate, a weak organic acid food preservative. Yeast 21(11): 927-946.

60. Kawahata M, Masaki K, Fujii T, Iefuji H (2006) Yeast genes involved in response to lactic acid and acetic acid: acidic conditions caused by the organic acids in Saccharomyces cerevisiae cultures induce expression of intracellular metal metabolism genes regulated by Aft1p. FEMS Yeast Res 6 (6): 924-936.

61. Jansen MLA, Bracher JM, Papapetridis I, Verhoeven MD, de Bruijn H, et al. (2017) Saccharomyces cerevisiae strains for second-generation ethanol production: from academic exploration to industrial implementation. FEMS Yeast Res 17(5): 1

62. Hou J, Qiu C, Shen Y, Li H, Bao X (2017) Engineering of Saccharomyces cerevisiae for the efficient co-utilization of glucose and xylose. FEMS Yeast Res 17 (4): 1-14.

63. Kim SR, Skerker JM, Kang W, Lesmana A, Wei N, et al. (2013) Rationa and Evolutionary Engineering Approaches Uncover a Small Set of Genetic Changes Efficient for Rapid Xylose Fermentation in Saccharomyces cerevisiae. PLoS One 8(2): e57048.

64. Zhang GC, Liu JJ, Kong II, Kwak S, Jin YS, et al. (2015) Combining C6 and C5 sugar metabolism for enhancing microbial bioconversion, Curr Opin Chem Biol 29 (2015) 49-57.

65. Sharma NK, Behera S, Arora R, Kumar S, Sani RK (2017) Xylose transport in yeast for lignocellulosic ethanol production: Current status. J Biosci Bioeng 125(3): 259-267.

66. Olsson L, Nielsen J (2000) The role of metabolic engineering in the improvement of Saccharomyces cerevisiae: Utilization of industrial media. Enzyme Microb Technol 26(9-10): 785-792.

67. Jeffries TW, Jin YS (2004) Metabolic engineering for improved fermentation of pentoses by yeasts. Appl Microbiol Biotechnol 63 (5): 495509. 
68. Hahn-Hägerdal B, Karhumaa K, Jeppsson M, Gorwa-Grauslund MF (2007) Metabolic engineering for pentose utilization in saccharomyces cerevisiae. Adv Biochem Eng Biotechnol 108: 147-177.

69. Hector RE, Dien BS, Cotta MA, Mertens JA (2013) Growth and fermentation of D-xylose by Saccharomyces cerevisiae expressing a novel D-xylose isomerase originating from the bacterium Prevotella ruminicola TC2-24. Biotechnol Biofuels 6 (1): 84.

70. Hahn-Hägerdal B, Galbe M, Gorwa-Grauslund MF, Lidén G, Zacchi G (2006) Bio-ethanol - the fuel of tomorrow from the residues of today. Trends Biotechnol 24 (12): 549-556.

71. Karhumaa K1, Garcia Sanchez R, Hahn-Hägerdal B, Gorwa-Grauslund MF (2007) Comparison of the xylose reductase-xylitol dehydrogenase and the xylose isomerase pathways for xylose fermentation by recombinant Saccharomyces cerevisiae. Microb Cell Fact 6: 5.

72. Brat D, Boles E, Wiedemann B (2009) Functional expression of a bacterial xylose isomerase in Saccharomyces cerevisiae. Appl Environ Microbiol 75 (8): 2304-2311.

73. Madhavan A, Tamalampudi S, Ushida K, Kanai D, Katahira S, et al. (2009) Xylose isomerase from polycentric fungus Orpinomyces: Gene sequencing, cloning, and expression in Saccharomyces cerevisiae for bioconversion of xylose to ethanol. Appl Microbiol Biotechnol 82 (6): 1067-1078.

74. de Figueiredo Vilela L, de Mello VM, Reis VC, Bon EP, Gonçalves Torres FA, et al. (2013) Functional expression of Burkholderia cenocepacia xylose isomerase in yeast increases ethanol production from a glucose-xylose blend. Bioresour Technol 128: 792-796.

75. Karhumaa K, Hahn-Hägerdal B, Gorwa-Grauslund MF (2005) Investigation of limiting metabolic steps in the utilization of xylose by recombinant Saccharomyces cerevisiae using metabolic engineering. Yeast 22 (5): 359-368.

76. Sonderegger M, Jeppsson M, Hahn-Hägerdal B, Sauer U (2004) Molecular Basis for Anaerobic Growth of Saccharomyces cerevisiae on Xylose, Investigated by Global Gene Expression and Metabolic Flux Analysis. Appl Environ Microbiol 70(4): 2307-2317.

77. Wahlbom CF, Cordero Otero RR, van Zyl WH, Hahn-Hägerdal B, Jönsson LJ (2003) Molecular analysis of a Saccharomyces cerevisiae mutant with improved ability to utilize xylose shows enhanced expression of proteins involved in transport, initial xylose metabolism, and the pentose phosphate pathway. Appl Environ Microbiol 69(2): 740-746.
78. Kruckeberg AL (1996) The hexose transporter family of Saccharomyces cerevisiae. Arch Microbiol 166(5): 283-292.

79. Lee WJ, Kim MD, Ryu YW, Bisson LF, Seo JH (2002) Kinetic studies on glucose and xylose transport in Saccharomyces cerevisiae. Appl Microbiol Biotechnol 60 (1-2): 186-191.

80. Young EM, Tong A, Bui H, Spofford C, Alper HS (2014) Rewiring yeast sugar transporter preference through modifying a conserved protein motif. Proc Natl Acad Sci 111 (1): 131-136.

81. Han JH, Park JY, Yoo KS, Kang HW, Choi GW, et al. (2011) Effect of glucose on xylose utilization in Saccharomyces cerevisiae harboring the xylose reductase gene. Arch Microbiol 193 (5): 335-340.

82. Leandro MJ, Gonçalves P, Spencer-Martins I (2006) Two glucose/xylose transporter genes from the yeast Candida intermedia: first molecular characterization of a yeast xylose-H+ symporter. Biochem J 395 (3): 543-549.

83. Runquist D, Fonseca C, Rådström P, Spencer-Martins I, Hahn-Hägerdal B (2009) Expression of the Gxf1 transporter from Candida intermedia improves fermentation performance in recombinant xylose-utilizing Saccharomyces cerevisiae. Appl Microbiol Biotechnol 82 (1): 123-130.

84. Wang C, Bao X, Li Y, Jiao C, Hou J, et al. (2015) Data set for cloning and characterization of heterologous transporters in Saccharomyces cerevisiae and identification of important amino acids for xylose utilization. Metab Eng 4: 119-126.

85. Ha SJ, Galazka JM, Kim SR, Choi JH, Yang X, et al. (2011) Engineered Saccharomyces cerevisiae capable of simultaneous cellobiose and xylose fermentation. Proc Natl Acad Sci U S A. 108 (2): 504-509.

86. Vilela Lde F, de Araujo VP, Paredes Rde S, Bon EP, Torres FA, et al. (2015) Enhanced xylose fermentation and ethanol production by engineered Saccharomyces cerevisiae strain. AMB Express 5: 16.

87. Salusjärvi L, Kankainen M, Soliymani R, Pitkänen JP, Penttilä M (2008) Ruohonen, Regulation of xylose metabolism in recombinant Saccharomyces cerevisiae. Microb Cell Fact 7:18.

88. Klimacek M, Krahulec S, Sauer U, Nidetzky B (2010) Nidetzky, Limitations in xylose-fermenting Saccharomyces cerevisiae, made evident through comprehensive metabolite profiling and thermodynamic analysis. Appl Environ Microbiol 76 (22): 7566-7574.

\section{Your next submission with Juniper Publishers will reach you the below assets}

- Quality Editorial service

- Swift Peer Review

- Reprints availability

- E-prints Service

- Manuscript Podcast for convenient understanding

- Global attainment for your research

- Manuscript accessibility in different formats

( Pdf, E-pub, Full Text, Audio)

- Unceasing customer service

Track the below URL for one-step submission

https://juniperpublishers.com/online-submission.php 